\title{
Powers of qualitative research
}

\author{
Old-fashioned qualitative research methods are still powerful in answering the most emergent climate questions \\ we are faced with.
}

\begin{abstract}
n natural and social science studies, quantitative methods emphasizing numbers and statistical or numerical models are becoming the dominant approach, and climate change research is no exception. In recent years, the scholarly community has witnessed a rapid rise in unprecedented data access and computational approaches. However, we should not ignore the powers of qualitative methods. Qualitative methods usually collect information through interviews and participant observation and from archival text. Rather than using large-scale datasets with common dimensions, they focus on unique cases and mechanisms ${ }^{1}$. In particular, they are inimitable when investigating underlying causes and data that are hard to measure or scarce.

With detailed descriptions of experience and opinions, qualitative methods are not only able to answer the 'what' questions, but also provide a better interpretation of the 'why' questions. In this month's issue, an Article by Kieran Findlater and colleagues discusses the underlying reason for why climate services fail to improve societal decision-making. Through interviews with
\end{abstract}

climate experts, the researchers find that the fundamental norms, institutions and governance of science need to be rethought. See a News \& Views from Meaghan Daly for further discussion of this issue.

Quantitative research relies on well-structured numbers. However, in the real world, evidence is often stored as descriptive language. Even with the growth of natural language processing (NLP) techniques ${ }^{2}$, qualitative text analysis is still essential to understand how climate social policies are designed or implemented. Two recent papers adopted such an approach. Through case-law analysis, Rupert Stuart-Smith and colleagues find that the scientific evidence submitted in climate-related cases is seriously behind the current frontier in climate science ${ }^{3}$. With both interpretative and inductive text analysis, Luis Zamarioli and colleagues examine the necessary condition of Article 2.1(c) that will facilitate consistency in climate finance flows ${ }^{4}$.

Over-reliance on quantitative research may enhance knowledge production inequality because maintaining large datasets often requires strong capacity. Since equity is one of the central issues in climate governance nowadays, field work and comparative case studies have irreplaceable roles in promoting a balanced climate research agenda. From empowering women's agency in climate responses in Asia and Africa ${ }^{5}$ to community-based adaptation initiatives in the Pacific Islands ${ }^{6}$, qualitative studies show their power in improving climate strategy in developing countries.

This is not to say that one research method is superior to another. In many cases, a mixed-methods strategy is necessary to answer the complex questions related to climate and society. Researchers should be open-minded to different approaches and focus on the questions that need to be answered.

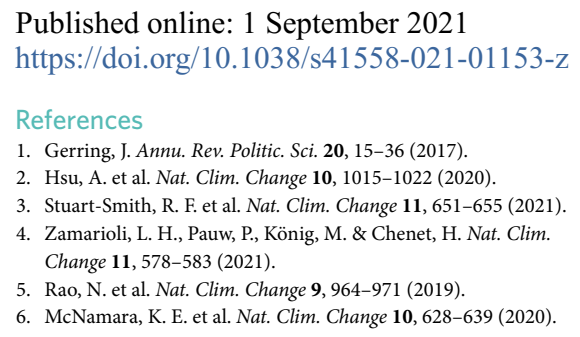

\section{Assessing the world today}

Climate extremes dominate headlines around the world as the IPCC releases its physical climate assessment report.

\footnotetext{
uly 2021 was the hottest month ever recorded (according to the US National Oceanic and Atmospheric Administration monthly global report ${ }^{1}$ ), with the global average surface temperature 0.93 ${ }^{\circ} \mathrm{C}$ above the twentieth century average. This puts 2021 on track to be one of the seven warmest years on record, pushing the planet ever close to exceeding the aspirational Paris Agreement $1.5^{\circ} \mathrm{C}$ warming limit. The recent IPCC Working Group 1 report $^{2}$ outlines how close we are to exceeding that limit - with the best estimate for human-caused warming approximately $1.1^{\circ} \mathrm{C}$ for $2010-2019$, relative to the 1850-1900 average. The report, and its headline statements, is a sombre read, but is ultimately unsurprising. There is now greater confidence - unequivocal on the global scale - in the effect of anthropogenic emissions on warming the planet and causing extreme events. And those events are occurring on a regular basis.
}

The droughts seen across the globe in the first half of the year ${ }^{3}$, and record heat, provided dry conditions for fires to take hold in July - areas around the Mediterranean burnt furiously, and the USA west coast again experienced some of their largest fires. Yet as fires rage, other regions, sometimes neighbouring countries, experience extreme rainfall and devastating floods. Images of roads turned into rivers and buildings being washed away alongside and yet contrasting with those of blackened skies and uncontrolled flames make all too clear our vulnerability to extreme climate events.

These images of infernos and floods are visually shocking, but the effects of climate change are everywhere, and not everything can be captured in this way. The recent report captures the extremes and their increase but also the unseen effects of climate change - improved estimates of climate sensitivity and changes in radiative forcing, as well as changes in atmospheric and ocean circulation, with implications for the hydrological cycle. This physical science report and the three Special reports released in this assessment cycle make it clear that aggressive action needs to start now to avoid the worst predicted effects. We hope that national and corporate leaders will respond now, before the next two sections Impacts, Adaptation and Vulnerability and Mitigation of Climate Change, due in early 2022, remind us that further time has slipped away.

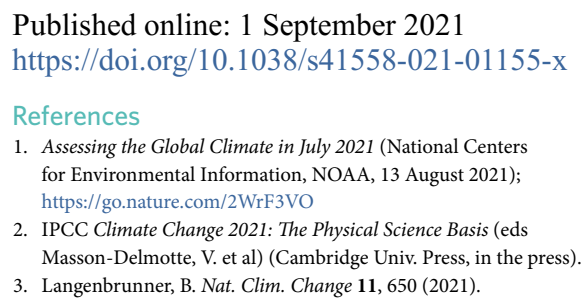

